\title{
Factors Associated with Having a Will, Power of Attorney, and Advanced Healthcare Directive in Patients Presenting to a Rural and Remote Memory Clinic
}

\author{
Sydney Lee (1), Andrew Kirk, Emily A. Kirk, Chandima Karunanayake, \\ Megan E. O'Connell, Debra Morgan
}

\begin{abstract}
Background: A Will, Power of Attorney, and Advanced Healthcare Directive are critical to guide decision-making in patients with dementia. We identified characteristics that are associated with the existence of these documents in patients who presented to a rural and remote memory clinic (RRMC). Methods: Ninety-five consecutive patients were included in this study. Patients and caregivers completed questionnaires on initial presentation to the RRMC and patients were asked if they had legal documents. Patients also completed neuropsychological testing. Statistical analysis ( $t$-test and $\chi^{2}$ test) was performed to identify significant variables. Results: Seventy (73.7\%) patients had a Will, 62 (65.3\%) had a Power of Attorney, and 21 (22.1\%) had an Advanced Healthcare Directive. Having a Will was associated with good quality of life ( $p=0.001)$, living alone or with a spouse or partner only $(p=0.034)$, poor verbal fluency $(p=0.055)$, and European ethnicity $(p=0.028)$. Factors associated with having a Power of Attorney included good quality of life $(p=0.031)$, living alone or with a spouse or partner only $(p=0.053)$, and poor verbal fluency $(p=0.015)$. Old age $(p=0.015)$, poor verbal fluency $(p=0.023)$, and greater severity of cognitive and functional impairment $(p=0.023)$ were associated with having an Advanced Healthcare Directive. Conclusions: Our results indicate that poor quality of life, good performance on verbal fluency, Indigenous ethnicity, and living with others are associated with a lower likelihood of legal documents in patients with dementia. These factors can help physicians identify patients at risk of leaving their legal affairs unattended to. Physicians should discuss the creation of legal documents early on in patients with signs of dementia.
\end{abstract}

RÉSUMÉ: Quels sont les facteurs associés au fait d'avoir un testament, une procuration et des directives médicales anticipées dans le cas de patients s'étant présentés à une clinique située en région rurale et éloignée ? Contexte: Un testament, une procuration et des directives médicales anticipées sont essentiels pour orienter la prise de décision dans le cas de patients atteints de démence. À cet égard, nous avons tenté d'identifier les caractéristiques associées à l'existence de ces documents légaux chez des patients s'étant présentés à une clinique située en région rurale et éloignée. Méthodes: Au total, 95 patients vus consécutivement ont été inclus dans cette étude. Ces patients, de même que leurs proches aidants, ont complété un questionnaire dès leur arrivée dans cette clinique. En plus de faire subir des tests neuropsychologiques aux patients, on leur a aussi demandé s'ils comptaient sur un des documents légaux cités précédemment. Pour identifier des variables significatives, nous avons effectué une analyse statistique au moyen des tests de Student et du $\chi^{2}$. Résultats: 70 patients $(73,7 \%)$ possédaient un testament ; $62(65,3 \%)$ avaient une procuration ; enfin, $21(22,1 \%)$ avaient établi des directives médicales anticipées. Le fait de posséder un testament a été associé à une bonne qualité de vie $(p=0,001)$, au fait de vivre seul ou en compagnie seulement d'un (e) conjoint (e) ou d'un (e) partenaire $(p=0,034)$, de montrer une faible fluidité verbale $(p=0,055)$ et de posséder des ancêtres d'origine européenne $(p=0,028)$. Maintenant, le fait de posséder une procuration a été associé aux facteurs suivants : une bonne qualité de vie ( $p=0,031)$, vivre seul ou en compagnie seulement d'un (e) conjoint (e) ou d'un (e) partenaire ( $p=0,053)$ et une faible fluidité verbale $(p=0,015)$. Enfin, ceux et celles ayant établi des directives médicales anticipées se sont révélés plus susceptibles d'être âgés $(p=0,015)$, d'être atteints d'une faible fluidité verbale $(p=0,023)$ et de montrer une déficience cognitive et fonctionnelle davantage prononcée $(p=0,023)$. Conclusions: Nos résultats indiquent qu'une piètre qualité de vie, qu'une élocution verbale aisée, que des origines autochtones et que le fait de partager sa vie avec plus d'une personne sont associés à une probabilité moindre de compter sur des documents légaux lorsqu'on est atteints de démence. Ces facteurs peuvent donc aider les médecins à repérer les patients à risque de négliger leurs affaires légales. À cet effet, les médecins devraient veiller à aborder très tôt la rédaction de ces documents avec des patients qui manifestent des symptômes de démence.

Keywords: Dementia, Memory, Will, Power of Attorney, Advanced Healthcare Directive doi:10.1017/cjn.2019.10

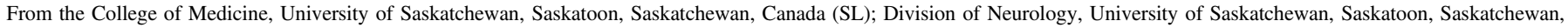

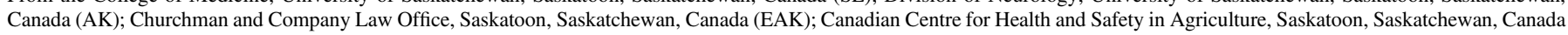
(CK, DM); Clinical Neuropsychology, University of Saskatchewan, Saskatoon, Saskatchewan, Canada (MOC)

Received August 19, 2018. Final Revisions Submitted December 27, 2018. Date of Acceptance January 18, 2019.

Correspondence to: Sydney Lee, College of Medicine, University of Saskatchewan, 107 Wiggins Road, Saskatoon, SK S7N 5E5, Canada. Email: sal052@mail.usask.ca 


\section{BACKGROUND}

Dementia is a broad category of neurological disease that leads to loss of independence, decreased quality of life, significant caregiver burden, and elevated healthcare costs. ${ }^{1}$ As Canada's population ages, the number of people living with dementia continues to increase. ${ }^{2}$ The Alzheimer Society of Canada estimated that in 2016, there were 564,000 persons in Canada living with dementia. It is predicted that this number will increase to 937,000 by $2031 .^{3}$ A number of cognitive functions are impaired in dementia including memory, orientation, language, and executive function. ${ }^{4,5} \mathrm{~A}$ matter of concern is the limited capacity of these individuals to take part in complex decisions regarding their financial and personal affairs. ${ }^{6}$ Questions regarding personal belongings and property, management of finances, and medical treatment may all arise when an individual is diagnosed with dementia. Legal documents such as a Will, Power of Attorney, and Advanced Healthcare Directive thus become critical to guide decision-making in these patients.

A Will is a legal document that provides instructions regarding the disposition of a person's estate. Making this document allows a person to devise, bequeath, or dispose of all real or personal property in which he or she has an interest at the time of his or her death. ${ }^{7}$ The document also allows one to appoint an executor to administer one's estate. Provincial legislation in each province governs the formation and validity of a Will.

The requirements for a valid Will are similar across most Canadian provinces. One necessary requirement for a Will is not found in legislation but is governed by common law: testamentary capacity. The four requirements for testamentary capacity are set out in the 1870 case of Banks $v$ Goodfellow. ${ }^{8}$ According to this case, the person creating a Will must understand the nature and effects of the act of making a Will, understand the extent of the property they are disposing of, comprehend and appreciate who will benefit from the estate, and be free from any delusions that may influence the making of the Will. ${ }^{8}$ Testamentary capacity can be challenged for legally defined reasons such as senility, insanity, delusions, and drugs or alcohol. It is important to note that although senility is still included in the legal literature, it is no longer a medical diagnosis and is not used in dementia literature.

"Senility" cases touch on issues that can arise for individuals with dementia. The Supreme Court of Canada recognizes that dementia patients can have a sliding scale of memory loss, and when a Will is made during one's illness, it is possible to have times of lucidity that do not necessarily invalidate the document. Testamentary capacity is important for the moment when instructions are given, and less so for the moment when the document is signed, as long as the person making the Will recalls having provided the instructions. ${ }^{9}$ Meanwhile, when someone with dementia becomes unable to meet the necessary elements of testamentary capacity, he or she is no longer able to create a valid Will.

It is important to create a Will in a timely manner, especially when an individual exhibits early signs of cognitive decline. If someone is unable to make a Will, their estate will, upon their death, be subject to the rules of intestacy. In this case, provincial estate administration legislation governs the way estates are dealt with. ${ }^{10}$ This legislation provides for a variety of familial situations and directs how the estate is to be divided.

A Power of Attorney is a document that allows a person to appoint another person to act on their behalf according to the terms of the document. As with the creation of a Will, provincial legislation in each province governs the creation of a Power of Attorney. The person creating the document, called the grantor, can appoint an attorney for property, for personal care, or for both. An attorney for property acts for the grantor in matters of property and financial affairs, whereas an attorney for personal care deals with the personal affairs of the grantor. ${ }^{11}$ A grantor must have capacity at the time of creating the document. A Power of Attorney can be contingent or enduring. If an enduring Power of Attorney is created, the power granted to the attorney is given to them at the time of the execution of the document. This authority will remain in place after the grantor loses capacity. ${ }^{11}$ If the Power of Attorney is contingent, it can be executed by the grantor while they have capacity, and the authority to act on their behalf will not vest in the attorney until the grantor loses capacity. $^{11}$

An Advanced Healthcare Directive is a document that gives instructions for healthcare decisions and/or the appointment of a substitute healthcare decision maker. In general, provincial legislation requires that the maker of the Advanced Healthcare Directive have capacity at the time of creating the directive. ${ }^{12}$ For instance, the person must have the ability to understand information relevant to a healthcare decision about a proposed treatment, appreciate the reasonably foreseeable consequences of making or not making a healthcare decision about a proposed treatment, and communicate a healthcare decision about a proposed treatment. ${ }^{12}$ It is standard for the directive to come into effect when a person loses capacity to make healthcare decisions.

As with a Will and Power of Attorney, capacity considerations are among reasons to have an Advanced Healthcare Directive prepared in an expedient manner. Should a patient fail to create an Advanced Healthcare Directive or appoint a substitute healthcare decision maker prior to losing capacity, alternate procedures for his or her healthcare decision-making are available. If possible, the nearest relative should make the decision on the patient's behalf. ${ }^{12}$ Beyond the nearest relative, each province sets out an ordered list of who is authorized to give consent to care on behalf of the patient.

Despite the importance of these documents, a large number of Canadians do not have them. Only $50.7 \%$ of Canadians aged 65 and older have an up-to-date Will, 29\% have a Power of Attorney, and $60 \%$ of hospitalized patients at high risk of dying have an Advanced Healthcare Directive. ${ }^{13-15}$ While these figures are a useful representation of the general population, there is no published data specific to patients with dementia.

Saskatchewan is a western Canadian province with a population of approximately 1 million. About $40 \%$ of this population lives in rural areas and small towns. ${ }^{16}$ These centers tend to have proportionally larger elderly populations than urban centers, with about one in five rural residents in Saskatchewan older than age $65 .{ }^{16}$ Along with the aging population elsewhere in Canada, these residents are at high risk of developing dementia. With a small population dispersed across a large province, geographical location is a critical barrier to accessing healthcare for people in rural communities. ${ }^{17}$ To improve access to specialized dementia care for the aging population in rural Saskatchewan, the Rural and Remote Memory Clinic (RRMC) was developed in 2004. At the initial presentation to the clinic, patients are assessed by a neurologist, neuropsychologist, physiotherapist, and dietitian. ${ }^{18}$ At the end of their visit, patients receive a diagnosis and a 
treatment plan. The clinic utilizes telehealth to follow-up with patients. This helps to limit the burden placed on caregivers and the healthcare system. ${ }^{19}$ More information on the RRMC is available elsewhere. $^{18-28}$

Decision-making is an increasingly important issue for patients with dementia. As the rates of dementia rise, more patients will experience irreversible cognitive incapacity and a loss of autonomy. ${ }^{2}$ The timely creation of a Will, Power of Attorney, and Advanced Healthcare Directive allows patients to participate in personal and medical decisions and alleviates stress from families. It remains unclear whether or not there are patient characteristics associated with having these documents. The objective of this study was to identify patient factors that are associated with having a Will, Power of Attorney, and Advanced Healthcare Directive in patients presenting to the RRMC. Identifying these factors may help physicians to better understand what makes patients more or less likely to have legal documents.

\section{Methods}

Ninety-five consecutive patients seen at the RRMC were included in this study. Sociodemographic and clinical variables were collected from questionnaires completed by patients and caregivers at the initial presentation to the RRMC. Variables include age, sex, ethnicity, years of formal education, education level, marital status, current housing, household members, and relationship of the caregiver to the patient. Cognition was screened with the Mini Mental Status Examination (MMSE). ${ }^{29}$ The Center for Epidemiologic Studies Depression Scale (CES-D) was used to screen for depression. ${ }^{30}$ Both patients and caregivers rated the patient's quality of life and ability to perform activities of daily living using the Quality of Life Scale (QOLS) and the Bristol Activities of Daily Living Scale (BADLS), respectively. ${ }^{31-33}$ Patients also completed the Instrumental Activities of Daily Living Scale (IADL). ${ }^{34}$ Caregiver-rated functional status of the patient was evaluated by the Functional Assessment Questionnaire (FAQ) and the severity component of the Neuropsychiatric Inventory Scale (NPI-S). ${ }^{35,36}$ The Clinical Dementia Rating Scale Sum of Boxes (CDR-SOB) was used to measure the severity of cognitive and functional impairment. ${ }^{32}$ Patients and their families were asked as part of their routine clinical assessment whether or not the patient had a Will, Power of Attorney, and/or an Advanced Healthcare Directive.

Neuropsychological testing included a 2-hour battery measuring the following cognitive domains: suboptimal performance, estimated premorbid verbal intelligence, expressive and receptive language, simple and complex attention, speed of mental processing, visuoperception/construction, memory, executive function, and social cognition. The battery results in over 100 possible scores, but for the sake of simplicity, we only present some of these scores with an emphasis on higher order cognitive functions. In particular, we include subtests from the Delis-Kaplan Executive Function System (D-KEFS) - specifically the Trail Making Test (Number-Letter Switching: Time-to-Completion), Verbal Fluency Test (Letter Fluency: Total Correct; Category Fluency: Total Correct; Category Switching: Total Correct Responses; Category Switching: Total Switching Accuracy), and Color-Word Interference Test (Inhibition: Time-to-Completion; Inhibition/Switching: Time-to-Completion). ${ }^{37}$ The Logical Memory I and II (immediate and delayed recall of a short story) subtest from Wechsler Memory Scale - Fourth Edition (WMS-IV) and California Verbal Learning Test - Second Edition (CVLT-II) Short-form (immediate recall, learning, free delayed recall, cued delayed recall, recognition) were used to assess memory. ${ }^{38,39}$ At the end of the initial clinic day, patients were given a diagnosis as agreed upon by the assessment team. Data from all 95 patients were analyzed for three separate analyses: the presence of a Will, Power of Attorney, and Advanced Healthcare Directive. For each analysis, patients were sorted into two groups based on whether or not they had the indicated document.

Data underwent statistical analysis using SPSS version $24 .^{40}$ Descriptive analyses were performed using frequencies, measures of central tendency, and measures of variance. For each of the three categories, the two groups (yes vs no) were compared using independent sample $t$-tests for continuous variables and $\chi^{2}$ for categorical variables. Exact test $p$ values were reported when expected values were small. Ethical approval was obtained from the University of Saskatchewan Behavioral Research Ethics Board.

\section{Results}

Ninety-five consecutive patients who underwent initial assessment at the RRMC were included in this study. Mean age was 70.84 years ( $\mathrm{SD}=10.18$ years) with a range of $43-90$ years and a median age of 72 . The sample comprised 46 males $(48.4 \%)$ and 49 females $(51.6 \%)$. The most frequent diagnosis was Alzheimer's disease $(N=34)$, followed by mild cognitive impairment $(N=28)$, frontotemporal dementia $(N=12)$, normal $(N=9)$, dementia due to other etiologies $(N=5)$, and vascular dementia $(N=2)$ (Tables 3, 6, and 9).

Seventy patients $(73.7 \%$ ) had a Will (Table 1 ). Patients who lived with others, aside from a spouse only, were significantly less likely to have a Will $(p=0.010)$. Patients who were accompanied to the clinic by someone other than an immediate family member were also less likely to have a Will $(p<0.0001)$. European ethnicity was significantly associated with having a Will ( $p=0.015)$. There was no statistically significant difference in age, sex, years of formal education, education level, marital status, or current housing between those who had a Will and those who did not. Patients with a better quality of life, as rated by the patient and the caregiver, were significantly more likely to have a Will (patient $p=0.001$; caregiver $p=0.055$ ). There was no significant association between having a Will and MMSE score, depressed mood, or patient function. Neuropsychological data revealed an association between a low verbal fluency performance and having a Will (category switching total [TTL] $p=0.055$; category switching accuracy $p=0.025 ; \%$ set loss errors $p=0.002$ ) (Table 1). Memory performance and severity of cognitive and functional impairment were not significantly associated with having a Will (Table 2). Likewise, there was no significant association between diagnosis (normal, mild cognitive impairment, frontotemporal dementia, Alzheimer's disease, vascular dementia, and dementia due to other etiologies) and having a Will (Table 3). The full comparison of patients with and without a Will is detailed in Tables 1-3.

Of the 95 patients in our sample, $62(65.3 \%)$ had a Power of Attorney (Table 4). Living with others, except for a spouse only, was significantly associated with not having a Power of Attorney $(p=0.038)$. There was no significant difference in age, sex, ethnicity, years of formal education, education level, marital status, 


\section{Table 1: Characteristics of patients with and without a Will*}

Variable

Age, in years $(n=95)$

Years of formal education $(n=81)$

MMSE, total score/30 $(n=78)$

Depressed Mood Scale (depression) $(n=77)$

Bristol Activities of Daily Living Scale $(n=41)$

Bristol Activities of Daily Living Scale - caregiver rated $(n=93)$

Quality of life of the patient as rated by the patient $(n=70)$

Quality of life of the patient as rated by caregiver $(n=86)$

Functional Assessment Questionnaire - caregiver rated $(n=92)$

Neuropsychiatric inventory-severity $(n=77)$

Instruments of daily living $(n=40)$

D-KEFS: Trail Making - Visual Scanning standard score tiTVSss $(n=44)$

D-KEFS: Trail Making - Number Sequencing standard score tiTNSss $(n=43)$

D-KEFS: Trail Making - Letter Sequencing standard score tiTLSss $(n=43)$

D-KEFS: Trail Making - Number-Letter Sequencing standard score tiTSWss $(n=38)$

D-KEFS: Trail Making - Motor Speed standard score tiTSss $(n=42)$

D-KEFS: Verbal Fluency - Letter Fluency standard score tiFLFss $(n=45)$

D-KEFS: Verbal Fluency - Category Fluency standard score tiFCFss $(n=43)$

D-KEFS: Verbal Fluency - Category Switching TTl standard score tiFSWTss $(n=42)$

D-KEFS: Verbal Fluency - Category Switching Acc standard score tiFSWAss $(n=43)$

D-KEFS: Verbal Fluency - \% Set loss errors standard score tiFSLss $(n=39)$

D-KEFS: Verbal Fluency - \% Repetition Errors standard score tiFREss $(n=39)$

D-KEFS: Stroop - Color Naming standard score tiSCNss $(n=46)$

D-KEFS: Stroop - Word Reading standard score tiSWRss $(n=46)$

D-KEFS: Stroop - inhibition standard score tiSIss $(n=44)$

D-KEFS: Stroop - Inhibition/Switching standard score tiSISss $(n=38)$

Sex $(n=95)$

Male

Female

Education level $(n=87)$

$<$ high school

$\geq$ high school

Marital status $(n=84)$

Married/common law

Other(single, divorced, separated, widowed)

Current housing $(n=84)$

Own house

Rented house/apartment

Other(special care, senior's high-rises, group home)

Lived with $(n=84)$

Alone

Spouse or partner only

Other(family members/friends, roommates, boarders)

\begin{tabular}{|c|c|c|c|}
\hline \multicolumn{3}{|c|}{ Will } & \multirow[b]{3}{*}{$p$ value by category } \\
\hline Yes $(n=70)$ & No $(n=25)$ & \multirow[b]{2}{*}{$p$ value } & \\
\hline Mean \pm SD & Mean \pm SD & & \\
\hline $71.71 \pm 9.63$ & $68.40 \pm 11.45$ & 0.164 & \\
\hline $11.72 \pm 2.80$ & $10.68 \pm 2.45$ & 0.153 & \\
\hline $24.78 \pm 3.58$ & $25.11 \pm 4.05$ & 0.739 & \\
\hline $11.90 \pm 7.76$ & $17.82 \pm 11.03$ & 0.014 & \\
\hline $3.38 \pm 5.62$ & $2.69 \pm 4.05$ & 0.746 & \\
\hline $6.00 \pm 7.33$ & $7.06 \pm 8.18$ & 0.554 & \\
\hline $38.59 \pm 4.87$ & $33.56 \pm 6.41$ & 0.001 & \\
\hline $33.89 \pm 6.39$ & $30.86 \pm 5.99$ & 0.055 & \\
\hline $10.87 \pm 6.90$ & $10.44 \pm 7.97$ & 0.802 & \\
\hline $7.87 \pm 5.47$ & $8.68 \pm 5.29$ & 0.556 & \\
\hline $24.72 \pm 3.24$ & $23.00 \pm 3.03$ & 0.135 & \\
\hline $7.75 \pm 3.77$ & $8.62 \pm 4.21$ & 0.563 & \\
\hline $7.43 \pm 4.65$ & $9.00 \pm 2.51$ & 0.200 & \\
\hline $6.77 \pm 4.41$ & $8.75 \pm 4.43$ & 0.259 & \\
\hline $5.26 \pm 4.11$ & $6.00 \pm 4.12$ & 0.669 & \\
\hline $9.91 \pm 2.52$ & $8.50 \pm 3.50$ & 0.193 & \\
\hline $7.33 \pm 3.61$ & $8.78 \pm 3.03$ & 0.276 & \\
\hline $6.76 \pm 3.77$ & $9.11 \pm 3.37$ & 0.098 & \\
\hline $5.76 \pm 3.64$ & $8.75 \pm 4.65$ & 0.055 & \\
\hline $5.57 \pm 3.25$ & $8.75 \pm 4.50$ & 0.025 & \\
\hline $7.68 \pm 4.33$ & $10.62 \pm 1.19$ & 0.002 & \\
\hline $9.39 \pm 3.79$ & $9.25 \pm 3.61$ & 0.927 & \\
\hline $7.39 \pm 3.92$ & $6.60 \pm 3.75$ & 0.573 & \\
\hline $8.17 \pm 3.69$ & $8.90 \pm 3.41$ & 0.576 & \\
\hline $7.26 \pm 4.10$ & $6.60 \pm 4.00$ & 0.653 & \\
\hline $6.82 \pm 4.06$ & $5.40 \pm 4.79$ & 0.371 & \\
\hline$n(\%)$ & $n(\%)$ & & \\
\hline 34 (48.6) & $12(48.0)$ & 0.961 & 0.960 \\
\hline $36(51.4)$ & $13(52.0)$ & & 0.960 \\
\hline $28(41.8)$ & $7(35.0)$ & 0.587 & 0.589 \\
\hline $39(58.2)$ & $13(65.0)$ & & 0.589 \\
\hline $48(73.8)$ & $11(57.9)$ & 0.181 & 0.180 \\
\hline $17(26.2)$ & $8(42.1)$ & & 0.180 \\
\hline $52(80.0)$ & $12(63.2)$ & 0.275 & 0.128 \\
\hline $6(9.2)$ & $4(21.1)$ & & 0.162 \\
\hline $7(10.8)$ & $3(15.8)$ & & 0.555 \\
\hline $15(23.1)$ & $2(10.5)$ & 0.034 & 0.230 \\
\hline $38(58.5)$ & $8(42.1)$ & & 0.208 \\
\hline $12(18.5)$ & $9(47.4)$ & & 0.010 \\
\hline
\end{tabular}


Table 1: (Continued)

\begin{tabular}{|c|c|c|c|c|}
\hline \multirow[b]{3}{*}{ Variable } & \multicolumn{3}{|c|}{ Will } & \multirow[b]{3}{*}{$p$ value by category } \\
\hline & Yes $(n=70)$ & No $(n=25)$ & \multirow[b]{2}{*}{$p$ value } & \\
\hline & Mean \pm SD & Mean \pm SD & & \\
\hline \multicolumn{5}{|c|}{ Respondent completing the questionnaire - Relationship to patient $(n=81)$} \\
\hline Wife & $20(32.8)$ & $4(20.0)$ & 0.001 & 0.276 \\
\hline Husband & $13(21.3)$ & $2(10.0)$ & & 0.258 \\
\hline Son & $7(11.5)$ & $2(10.0)$ & & 0.857 \\
\hline Daughter & $19(31.1)$ & $4(20.0)$ & & 0.337 \\
\hline Other & $2(3.3)$ & $8(40.0)$ & & $<0.0001$ \\
\hline \multicolumn{5}{|c|}{ Ethnicity $(n=82)$} \\
\hline European & 59 (93.7) & $14(73.7)$ & 0.028 & 0.015 \\
\hline Indigenous & $4(6.3)$ & $5(26.3)$ & & 0.015 \\
\hline
\end{tabular}

*All variables have missing values except age and sex.

\section{Table 2: Memory and severity characteristics of patients with and without a Will}

\begin{tabular}{|c|c|c|c|}
\hline \multirow[b]{3}{*}{ Variable } & \multicolumn{2}{|c|}{ Will } & \multirow[b]{3}{*}{$p$ value } \\
\hline & Yes $(n=70)$ & No $(n=25)$ & \\
\hline & Mean \pm SD & Mean \pm SD & \\
\hline WMS-IV: LM I standard score $(n=47)$ & $7.73 \pm 11.02$ & $5.10 \pm 2.33$ & 0.461 \\
\hline WMS-IV: LM II standard score $(n=47)$ & $4.22 \pm 3.45$ & $4.30 \pm 2.16$ & 0.942 \\
\hline CVLT-II Short: total trials $1-4$ standard score $(n=46)$ & $36.92 \pm 12.00$ & $36.30 \pm 9.45$ & 0.882 \\
\hline CVLT-II Short: short delay free recall standard score $(n=46)$ & $-1.36 \pm 1.31$ & $-1.15 \pm 0.71$ & 0.628 \\
\hline CVLT-II Short: long delay free recall standard score $(n=46)$ & $-1.38 \pm 1.11$ & $-1.35 \pm 0.82$ & 0.948 \\
\hline CVLT-II Short: long delay cued recall standard score $(n=46)$ & $-1.26 \pm 1.34$ & $-1.65 \pm 1.08$ & 0.407 \\
\hline CVLT-II Short: total intrusions standard score $(n=46)$ & $1.96 \pm 1.87$ & $2.10 \pm 1.72$ & 0.831 \\
\hline CVLT-II Short: recognition hits standard score $(n=45)$ & $-1.09 \pm 1.21$ & $-0.85 \pm 1.43$ & 0.605 \\
\hline CVLT-II Short: recognition FP standard score $(n=46)$ & $1.74 \pm 1.61$ & $2.65 \pm 1.51$ & 0.116 \\
\hline CDR-SOB (severity) $(n=84)$ & $3.85 \pm 2.58$ & $3.93 \pm 3.16$ & 0.901 \\
\hline
\end{tabular}

current housing, or relationship of caregiver to the patient between patients who had a Power of Attorney and those who did not. Patients who reported a better quality of life were significantly more likely to have a Power of Attorney (patient $p=0.031$; caregiver $p=0.050$ ). MMSE, depressed mood, and patient function were not significantly associated with having a Power of Attorney. Lower scores on visual scanning, number sequencing, and letter sequencing were significantly associated with having a Power of Attorney (visual scanning $p=0.009$; number sequencing $p=0.010$; letter sequencing $p=0.045$ ). Patients with lower scores on verbal fluency were significantly more likely to have a Power of Attorney (category fluency $p=0.015$; category switching accuracy $p=0.053$ ) (Table 4). Measures of memory and severity (Table 5) as well as diagnostic category (Table 6) were not significant. The full comparison of patients with and without a Power of Attorney is presented in Tables 4-6.

The majority of patients did not have an Advanced Healthcare Directive, with only 21 patients $(22.1 \%)$ in the "yes" group
(Table 7). Patients who had an Advanced Healthcare Directive were significantly older than those who did not $(p=0.015)$. Living in housing categorized as "other," such as special care, senior's centers, and group homes was significantly associated with having an Advanced Healthcare Directive $(p=0.038)$. There was no association with sex, ethnicity, years of formal education, education level, marital status, relationship of the caregiver to the patient, or with whom the patient lived. Patients with a lower level of caregiver-rated function and independence were significantly more likely to have an Advanced Healthcare Directive $(p=0.047)$. MMSE, depressed mood, and patient quality of life were not significant. Poor verbal fluency was significantly associated with having an Advanced Healthcare Directive (category switching TTI $p=0.023$; category switching accuracy $p=0.004)$. Other neuropsychological measures including memory were not significant (Table 7). Patients with greater severity of cognitive and functional impairment as measured by CDR-SOB were significantly more likely to have an 
Table 3: Neurological diagnosis of patients with and without a Will

\begin{tabular}{l|c|c|c|c|}
\hline \multirow{2}{*}{ Diagnosis } & \multicolumn{3}{|c|}{ Will } & Total $(\boldsymbol{n}=\mathbf{9 0})$ \\
\cline { 2 - 5 } & Yes $(\boldsymbol{n}=\mathbf{6 7})$ & No $(\boldsymbol{n}=\mathbf{2 3})$ & $\boldsymbol{n}(\boldsymbol{\%})$ & $\boldsymbol{p}$ value by category \\
\cline { 2 - 5 } Normal & $\boldsymbol{n}(\boldsymbol{\%})$ & $\boldsymbol{n}(\boldsymbol{\%})$ & $9(10.0)$ & 0.575 \\
\hline Mild cognitive impairment & $6(9.0)$ & $3(13.0)$ & $28(31.1)$ & 0.139 \\
\hline Frontotemporal dementia & $18(26.9)$ & $10(43.5)$ & $12(13.3)$ & 0.447 \\
\hline Alzheimer's disease & $10(14.9)$ & $2(8.7)$ & $2(37.8)$ & 0.180 \\
\hline Vascular dementia & $28(41.8)$ & $1(26.1)$ & $5(5.3)$ & 0.424 \\
\hline Dementia due to other etiologies & $1(1.5)$ & $1(4.3)$ & & 0.772 \\
\hline
\end{tabular}

Table 4: Characteristics of patients with and without a Power of Attorney*

\begin{tabular}{|c|c|c|c|c|}
\hline \multirow[b]{3}{*}{ Variable } & \multicolumn{2}{|c|}{ Power of Attorney } & \multirow[b]{3}{*}{$p$ value } & \multirow[b]{3}{*}{$p$ value by category } \\
\hline & Yes $(n=62)$ & No $(n=33)$ & & \\
\hline & Mean \pm SD & Mean \pm SD & & \\
\hline Age, in years $(n=95)$ & $71.58 \pm 9.28$ & $69.45 \pm 11.72$ & 0.335 & \\
\hline Years of formal education $(n=81)$ & $11.64 \pm 2.73$ & $11.11 \pm 2.80$ & 0.421 & \\
\hline MMSE, total score/30 $(n=78)$ & $24.65 \pm 3.51$ & $25.27 \pm 4.02$ & 0.489 & \\
\hline Depressed Mood Scale (depression) $(n=77)$ & $12.20 \pm 7.92$ & $15.57 \pm 10.58$ & 0.128 & \\
\hline Bristol Activities of Daily Living Scale $(n=41)$ & $3.82 \pm 6.12$ & $2.14 \pm 3.13$ & 0.346 & \\
\hline Bristol Activities of Daily Living Scale - caregiver rated $(n=93)$ & $5.83 \pm 7.25$ & $7.16 \pm 8.10$ & 0.425 & \\
\hline Quality of life of the patient as rated by the patient $(n=70)$ & $38.33 \pm 5.11$ & $35.50 \pm 6.31$ & 0.031 & \\
\hline Quality of life of the patient as rated by caregiver $(n=86)$ & $34.18 \pm 6.46$ & $31.03 \pm 5.84$ & 0.050 & \\
\hline Functional Assessment Questionnaire-caregiver rated $(n=92)$ & $10.70 \pm 6.78$ & $10.84 \pm 7.96$ & 0.928 & \\
\hline Neuropsychiatric inventory-severity $(n=77)$ & $7.31 \pm 4.98$ & $9.50 \pm 5.88$ & 0.086 & \\
\hline Instruments of daily living $(n=40)$ & $24.71 \pm 3.29$ & $23.17 \pm 2.95$ & 0.169 & \\
\hline D-KEFS: Trail Making - Visual Scanning standard score tiTVSss $(n=44)$ & $7.03 \pm 4.02$ & $9.78 \pm 2.55$ & 0.009 & \\
\hline D-KEFS: Trail Making - Number Sequencing standard score tiTNSss $(n=43)$ & $6.76 \pm 4.75$ & $9.71 \pm 2.46$ & 0.010 & \\
\hline D-KEFS: Trail Making - Letter Sequencing standard score tiTLSss $(n=43)$ & $6.21 \pm 4.24$ & $9.07 \pm 4.30$ & 0.045 & \\
\hline $\begin{array}{l}\text { D-KEFS: Trail Making - Number-Letter Sequencing standard score tiTSWss } \\
(n=38)\end{array}$ & $4.58 \pm 3.94$ & $7.17 \pm 3.90$ & 0.067 & \\
\hline D-KEFS: Trail Making - Motor Speed standard score tiTSss $(n=42)$ & $9.14 \pm 2.92$ & $10.64 \pm 2.09$ & 0.065 & \\
\hline D-KEFS: Verbal Fluency - Letter Fluency standard score tiFLFss $(n=45)$ & $6.97 \pm 3.69$ & $8.93 \pm 2.81$ & 0.077 & \\
\hline D-KEFS: Verbal Fluency - Category Fluency standard score tiFCFss $(n=43)$ & $6.25 \pm 3.75$ & $9.13 \pm 3.14$ & 0.015 & \\
\hline $\begin{array}{l}\text { D-KEFS: Verbal Fluency - Category Switching TTl standard score tiFSWTss } \\
(n=42)\end{array}$ & $5.57 \pm 3.65$ & $7.86 \pm 4.28$ & 0.079 & \\
\hline $\begin{array}{l}\text { D-KEFS: Verbal Fluency - Category Switching Acc standard score tiFSWAss } \\
(n=43)\end{array}$ & $5.41 \pm 3.33$ & $7.71 \pm 3.97$ & 0.053 & \\
\hline D-KEFS: Verbal Fluency $-\%$ set loss errors standard score tiFSLss $(n=39)$ & $7.68 \pm 4.39$ & $9.36 \pm 3.27$ & 0.221 & \\
\hline $\begin{array}{l}\text { D-KEFS: Verbal Fluency - \% repetition errors standard score tiFREss } \\
(n=39)\end{array}$ & $9.28 \pm 3.90$ & $9.50 \pm 3.48$ & 0.862 & \\
\hline D-KEFS: Stroop - Color Naming standard score tiSCNss $(n=46)$ & $6.93 \pm 4.10$ & $7.75 \pm 3.41$ & 0.500 & \\
\hline D-KEFS: Stroop - Word Reading standard score tiSWRss $(n=46)$ & $7.87 \pm 3.58$ & $9.19 \pm 3.62$ & 0.241 & \\
\hline D-KEFS: Stroop - Inhibition standard score tiSIss $(n=44)$ & $6.59 \pm 4.20$ & $8.13 \pm 3.64$ & 0.234 & \\
\hline D-KEFS: Stroop - Inhibition/Switching standard score tiSISss $(n=38)$ & $5.56 \pm 3.89$ & $7.80 \pm 4.54$ & 0.114 & \\
\hline
\end{tabular}


Table 4: (Continued)

\begin{tabular}{|c|c|c|c|c|}
\hline \multirow[b]{3}{*}{ Variable } & \multicolumn{2}{|c|}{ Power of Attorney } & \multirow[b]{3}{*}{$p$ value } & \multirow[b]{3}{*}{$p$ value by category } \\
\hline & Yes $(n=62)$ & No $(n=33)$ & & \\
\hline & Mean \pm SD & Mean \pm SD & & \\
\hline $\operatorname{Sex}(n=95)$ & $n(\%)$ & $n(\%)$ & & \\
\hline Male & $27(43.5)$ & $19(41.3)$ & 0.193 & 0.194 \\
\hline Female & $35(71.4)$ & $14(28.6)$ & & 0.194 \\
\hline \multicolumn{5}{|l|}{ Education level $(n=87)$} \\
\hline$<$ high school & $26(43.3)$ & $9(33.3)$ & 0.379 & 0.379 \\
\hline$\geq$ high school & $34(56.7)$ & $18(66.7)$ & & 0.379 \\
\hline \multicolumn{5}{|l|}{ Marital status $(n=84)$} \\
\hline Married/common law & $41(70.7)$ & $18(69.2)$ & 0.892 & 0.889 \\
\hline Other(single, divorced, separated, widowed) & $17(29.3)$ & $8(30.8)$ & & 0.889 \\
\hline \multicolumn{5}{|l|}{ Current housing $(n=84)$} \\
\hline Own house & $45(76.2)$ & $19(76.0)$ & 1.000 & 0.976 \\
\hline Rented house/apartment & $7(11.9)$ & $3(12.0)$ & & 0.984 \\
\hline Other(special care, senior's high-rises, group home) & $7(11.9)$ & $3(12.0)$ & & 0.984 \\
\hline \multicolumn{5}{|l|}{ Lived with $(n=84)$} \\
\hline Alone & $15(25.4)$ & $2(8.0)$ & 0.053 & 0.069 \\
\hline Spouse or partner only & $33(55.9)$ & $13(52.0)$ & & 0.741 \\
\hline Other(family members/friends, roommates, boarders) & 11 (18.6) & $10(40.0)$ & & 0.038 \\
\hline \multicolumn{5}{|c|}{ Respondent completing the questionnaire - relationship to patient $(n=81)$} \\
\hline Wife & $17(30.4)$ & $7(28.0)$ & 0.072 & 0.834 \\
\hline Husband & $12(21.4)$ & $3(12.0)$ & & 0.312 \\
\hline Son & $7(12.5)$ & $2(8.0)$ & & 0.548 \\
\hline Daughter & $17(30.4)$ & $6(24.0)$ & & 0.555 \\
\hline Other & $3(5.4)$ & $7(28.0)$ & & 0.141 \\
\hline \multicolumn{5}{|l|}{ Ethnicity $(n=82)$} \\
\hline European & $52(92.9)$ & $21(80.8)$ & 0.103 & 0.103 \\
\hline Indigenous & $4(7.1)$ & $5(19.2)$ & & 0.103 \\
\hline
\end{tabular}

*All variables have missing values except age and sex.

Advanced Healthcare Directive ( $p=0.023$ ) (Table 8). There was no significant association between having an Advanced Healthcare Directive and diagnosis (Table 9). The full comparison of patients with and without an Advanced Healthcare Directive is outlined in Tables 7-9.

\section{Discussion}

Decision-making requires many of the cognitive domains that are compromised in dementia. ${ }^{5,6}$ Legal documents such as a Will, Power of Attorney, and Advanced Healthcare Directive become critical for patients who develop significant deficits in cognition. It is unknown whether there exist patient factors that might contribute to a greater likelihood of procuring these documents. In order to better address issues of decision-making and cognitive capacity in patients with dementia, we sought to identify trends in their clinical and demographic characteristics. This may help to recognize patients who are at greater risk of not having legal documents and those who are likely to benefit from further attention to legal planning.
Our data revealed similarities between patients who had a Will and patients who had a Power of Attorney. With whom patients lived was a strong clinical indicator. Patients who lived with others, excluding a spouse or partner only, were less likely to have a Will and Power of Attorney. One explanation for this may be that patients who live with family members, friends, roommates, or boarders do not have ownership or are not the sole owners of the property where they are living, thus decreasing the need to create a Will. Compared to living alone or with a spouse of similar functional capacity, perhaps living with others provides patients with more opportunities to have help with managing their finances, therefore lessening the perceived need for a Power of Attorney. Patients who were accompanied to the clinic by someone other than an immediate family member were less likely to have a Will. Interestingly, older age was not significantly associated with having either a Will or a Power of Attorney.

We found that patients with a Will and/or Power of Attorney tended to have a better quality of life as rated by both the patient 
Table 5: Memory and severity characteristics of patients with and without a Power of Attorney

\begin{tabular}{l|c|c|c|}
\hline \multirow{2}{*}{ Variable } & Yes $(\boldsymbol{n}=\mathbf{6 2})$ & Power of Attorney & No $(\boldsymbol{n}=\mathbf{3 3})$ \\
\cline { 2 - 4 } & Mean \pm SD & Mean \pm SD & \multicolumn{1}{c}{$\boldsymbol{p}$ value } \\
\cline { 2 - 4 } WMS-IV: LM I standard score $(n=47)$ & $7.87 \pm 12.06$ & $5.81 \pm 2.14$ & 0.504 \\
\hline WMS-IV: LM II standard score $(n=47)$ & $3.94 \pm 3.60$ & $4.81 \pm 2.23$ & 0.379 \\
\hline CVLT-II Short: total trials $1-4$ standard score $(n=46)$ & $35.3 \pm 11.77$ & $39.5 \pm 10.48$ & 0.242 \\
\hline CVLT-II Short: short-delay free recall standard score $(n=46)$ & $-1.50 \pm 1.17$ & $-0.97 \pm 1.22$ & 0.154 \\
\hline CVLT-II Short: long-delay free recall standard score $(n=46)$ & $-1.52 \pm 1.10$ & $-1.09 \pm 0.90$ & 0.195 \\
\hline CVLT-II Short: long-delay cued recall standard score $(n=46)$ & $-1.55 \pm 1.28$ & $-0.97 \pm 1.26$ & 0.146 \\
\hline CVLT-II Short: total intrusions standard score $(n=46)$ & $2.15 \pm 2.04$ & $1.69 \pm 1.31$ & 0.358 \\
\hline CVLT-II Short: recognition hits standard score $(n=45)$ & $-1.07 \pm 1.22$ & $-0.97 \pm 1.35$ & 0.800 \\
\hline CVLT-II Short: recognition FP standard score $(n=46)$ & $2.12 \pm 1.64$ & $1.59 \pm 1.58$ & 0.303 \\
\hline CDR-SOB (severity) $(n=84)$ & $3.79 \pm 2.55$ & $4.02 \pm 3.06$ & \\
\hline
\end{tabular}

Table 6: Neurological diagnosis of patients with and without a Power of Attorney

\begin{tabular}{|c|c|c|c|c|}
\hline \multirow[b]{3}{*}{ Diagnosis } & \multicolumn{3}{|c|}{ Power of Attorney } & \multirow[b]{2}{*}{$p$ value by category } \\
\hline & Yes $(n=59)$ & No $(n=31)$ & Total $(n=90)$ & \\
\hline & $n(\%)$ & $n(\%)$ & $n(\%)$ & \multirow[b]{2}{*}{0.161} \\
\hline Normal & $4(6.8)$ & $5(16.1)$ & $9(10.0)$ & \\
\hline Mild cognitive impairment & $17(28.8)$ & $11(35.5)$ & $28(31.1)$ & 0.516 \\
\hline Frontotemporal dementia & $9(15.3)$ & $3(9.7)$ & $12(13.3)$ & 0.459 \\
\hline Alzheimer's disease & $24(40.7)$ & $10(32.3)$ & $34(37.8)$ & 0.435 \\
\hline Vascular dementia & $1(1.7)$ & $1(3.2)$ & $2(2.2)$ & 0.638 \\
\hline Dementia due to other etiologies & $4(6.8)$ & $1(3.2)$ & $5(5.6)$ & 0.484 \\
\hline
\end{tabular}

and caregiver. It is possible that those with a better quality of life are more impacted by physical and cognitive changes as they age and thus become more aware of the need for a Will or Power of Attorney. The QOLS used to rate quality of life in this study considers factors such as energy level, living situation, family, and money. ${ }^{31}$ Patients who are lacking in any of these areas may experience more difficulty scheduling and keeping an appointment to create a Will or Power of Attorney, or they may not have the finances to do so. Physicians should discuss finances and the ability to execute a plan with patients when recommending that they create legal documents.

A common pattern emerged among patients with a Will, patients with a Power of Attorney, and patients with an Advanced Healthcare Directive. In all three groups, poor verbal fluency was associated with having the specified document. Verbal fluency tasks require patients to retrieve words from their mental lexicon, focus on the task, select words meeting certain constraints, and avoid repetition. ${ }^{41}$ Thus, deficits in either verbal ability or executive control manifest themselves in poor performance on the fluency tasks. ${ }^{42}$ Although it is common to experience wordfinding difficulties with normal aging, more profound deficits in verbal ability may indicate cognitive impairment. ${ }^{43}$ It may be that noticeable problems with verbal fluency, unlike other cognitive deficits, spur family members to recognize there is a problem and to advocate for the completion of legal documents. Interestingly, measures of memory were not strongly associated with having any of the three legal documents. In contrast to language difficulties, patients may be more able to hide their memory problems in the early stages of dementia. Compensatory strategies used by patients with memory loss may include avoiding tasks that they no longer remember how to perform, relying on their spouse to fill in memory gaps or inventing stories to excuse lapses in memory. ${ }^{44}$ Caregivers and family members may fail to recognize changes in the patient's memory or attribute memory problems to normal aging. ${ }^{45,46}$

Our data revealed that lower scores on Trail Making tasks were associated with having a Power of Attorney. Trail Making tasks reflect a wide variety of cognitive processes including simple and complex attention, visual search and scanning, psychomotor integration, sequencing and shifting, flexibility, ability to execute and modify a plan of action, and ability to maintain two trains of thought simultaneously. ${ }^{47}$ It is possible that many of these cognitive functions are involved in managing finances or maintaining a budget. Thus, patients who demonstrate poor performance on Trail Making tasks may also have an increased need for a Power of Attorney. 


\section{Table 7: Characteristics of patients with and without an Advanced Healthcare Directive*}

Variable

Age, in years $(n=95)$

Years of formal education $(n=81)$

MMSE, total score/30 $(n=78)$

Depressed Mood Scale (depression) $(n=77)$

Bristol Activities of Daily Living Scale $(n=41)$

Bristol Activities of Daily Living Scale - caregiver rated $(n=93)$

Quality of life of the patient as rated by the patient $(n=70)$

Quality of life of the patient as rated by caregiver $(n=86)$

Functional Assessment Questionnaire - caregiver rated $(n=92)$

Neuropsychiatric inventory - severity $(n=77)$

Instruments of daily living $(n=40)$

D-KEFS: Trail Making - Visual Scanning standard score tiTVSss $(n=44)$

D-KEFS: Trail Making - Number Sequencing standard score tiTNSss $(n=43)$

D-KEFS: Trail Making - Letter Sequencing standard score tiTLSss $(n=43)$

D-KEFS: Trail Making - Number-Letter Sequencing standard score tiTSWss $(n=38)$

D-KEFS: Trail Making - Motor Speed standard score tiTSss $(n=42)$

D-KEFS: Verbal Fluency - Letter Fluency standard score tiFLFss $(n=45)$

D-KEFS: Verbal Fluency - Category Fluency standard score tiFCFss $(n=43)$

D-KEFS: Verbal Fluency - Category Switching TTl standard score tiFSWTss $(n=42)$

D-KEFS: Verbal Fluency - Category Switching Acc standard score tiFSWAss $(n=43)$

D-KEFS: Verbal Fluency - \% set loss errors standard score tiFSLss $(n=39)$

D-KEFS: Verbal Fluency - \% repetition errors standard score tiFREss $(n=39)$

D-KEFS: Stroop - Color Naming standard score tiSCNss $(n=46)$

D-KEFS: Stroop - Word Reading standard score tiSWRss $(n=46)$

D-KEFS: Stroop - Inhibition standard score tiSIss $(n=44)$

D-KEFS: Stroop - Inhibition/switching standard score tiSISss $(n=38)$

Sex $(n=95)$

Male

Female

Education level $(n=87)$

$<$ high school

$\geq$ high school

Marital status $(n=84)$

Married/common law

Other(single, divorced, separated, widowed)

Current housing $(n=84)$

Own house

Rented house/apartment

Other (special care, senior's high-rises, group home)

Lived with $(n=84)$

Alone

Spouse or partner only

Other(family members/friends, roommates, boarders)

\begin{tabular}{|c|c|c|c|}
\hline \multicolumn{2}{|c|}{ Advanced Healthcare Directive } & \multirow[b]{3}{*}{$p$ value } & \multirow[b]{3}{*}{$p$ value by category } \\
\hline Yes $(n=21)$ & No $(n=74)$ & & \\
\hline Mean \pm SD & Mean \pm SD & & \\
\hline $75.57 \pm 8.83$ & $69.50 \pm 10.19$ & 0.015 & \\
\hline $11.61 \pm 3.36$ & $11.44 \pm 2.57$ & 0.814 & \\
\hline $24.17 \pm 3.71$ & $25.07 \pm 3.67$ & 0.365 & \\
\hline $13.63 \pm 10.14$ & $13.07 \pm 8.49$ & 0.812 & \\
\hline $6.81 \pm 8.93$ & $2.38 \pm 3.72$ & 0.208 & \\
\hline $9.14 \pm 9.01$ & $5.44 \pm 6.89$ & 0.047 & \\
\hline $38.00 \pm 5.69$ & $37.28 \pm 5.65$ & 0.655 & \\
\hline $31.38 \pm 7.04$ & $33.68 \pm 6.13$ & 0.154 & \\
\hline $13.38 \pm 6.96$ & $9.97 \pm 7.09$ & 0.055 & \\
\hline $8.33 \pm 5.57$ & $8.03 \pm 5.39$ & 0.838 & \\
\hline $25.10 \pm 2.18$ & $23.97 \pm 3.51$ & 0.345 & \\
\hline $7.30 \pm 4.19$ & $8.09 \pm 3.74$ & 0.572 & \\
\hline $8.20 \pm 7.57$ & $5.29 \pm 4.11$ & 0.696 & \\
\hline $6.10 \pm 4.79$ & $7.45 \pm 4.34$ & 0.403 & \\
\hline $5.33 \pm 4.21$ & $5.41 \pm 4.09$ & 0.959 & \\
\hline $9.50 \pm 3.34$ & $9.69 \pm 2.58$ & 0.853 & \\
\hline $8.33 \pm 5.15$ & $7.44 \pm 3.06$ & 0.504 & \\
\hline $7.50 \pm 4.75$ & $7.20 \pm 3.59$ & 0.842 & \\
\hline $3.50 \pm 3.25$ & $7.00 \pm 3.87$ & 0.023 & \\
\hline $3.11 \pm 2.52$ & $6.97 \pm 3.52$ & 0.004 & \\
\hline $6.14 \pm 5.14$ & $8.75 \pm 3.72$ & 0.126 & \\
\hline $11.00 \pm 2.00$ & $9.00 \pm 3.92$ & 0.200 & \\
\hline $7.27 \pm 4.75$ & $7.20 \pm 3.61$ & 0.957 & \\
\hline $7.82 \pm 4.47$ & $8.48 \pm 3.35$ & 0.598 & \\
\hline $6.80 \pm 4.73$ & $7.20 \pm 3.89$ & 0.784 & \\
\hline $6.33 \pm 3.78$ & $6.47 \pm 4.38$ & 0.944 & \\
\hline$n(\%)$ & $n(\%)$ & & \\
\hline $11(52.4)$ & $35(47.3)$ & 0.681 & 0.681 \\
\hline $10(47.6)$ & $39(52.7)$ & & 0.681 \\
\hline $8(40.0)$ & $27(40.3)$ & 0.981 & 0.984 \\
\hline $12(60.0)$ & $40(59.7)$ & & 0.984 \\
\hline $12(60.0)$ & $47(73.4)$ & 0.251 & 0.250 \\
\hline $8(40.0)$ & $17(26.6)$ & & 0.250 \\
\hline $14(70.0)$ & $50(78.1)$ & 0.084 & 0.459 \\
\hline $1(5.0)$ & $9(14.1)$ & & 0.276 \\
\hline $5(25.0)$ & $5(7.8)$ & & 0.038 \\
\hline $6(30.0)$ & $11(17.2)$ & 0.429 & 0.215 \\
\hline $9(45.0)$ & $37(57.8)$ & & 0.317 \\
\hline $5(25.0)$ & $16(25.0)$ & & 1.000 \\
\hline
\end{tabular}


Table 7: (Continued)

\begin{tabular}{|c|c|c|c|c|}
\hline \multirow[b]{3}{*}{ Variable } & \multicolumn{2}{|c|}{ Advanced Healthcare Directive } & \multirow[b]{3}{*}{$p$ value } & \multirow[b]{3}{*}{$p$ value by category } \\
\hline & Yes $(n=21)$ & No $(n=74)$ & & \\
\hline & Mean \pm SD & Mean \pm SD & & \\
\hline \multicolumn{5}{|c|}{ Respondent completing the questionnaire - relationship to patient $(n=81)$} \\
\hline Wife & $5(26.3)$ & $19(30.6)$ & 0.310 & 0.719 \\
\hline Husband & $2(10.5)$ & $13(21.0)$ & & 0.303 \\
\hline Son & $4(21.1)$ & $5(8.1)$ & & 0.114 \\
\hline Daughter & $7(36.8)$ & $16(25.8)$ & & 0.352 \\
\hline Other & $1(5.3)$ & $9(14.5)$ & & 0.285 \\
\hline \multicolumn{5}{|c|}{ Ethnicity $(n=82)$} \\
\hline European & $18(90.0)$ & $55(88.7)$ & 0.872 & 0.873 \\
\hline Indigenous & $2(10.0)$ & $7(11.3)$ & & 0.873 \\
\hline
\end{tabular}

*All variables have missing values except age and sex.

Table 8: Memory and severity characteristics of patients with and without an Advanced Healthcare Directive

\begin{tabular}{|c|c|c|c|}
\hline \multirow[b]{3}{*}{ Variable } & \multicolumn{2}{|c|}{ Advanced Healthcare Directive } & \multirow[b]{3}{*}{$p$ value } \\
\hline & Yes $(n=21)$ & No $(n=74)$ & \\
\hline & Mean \pm SD & Mean \pm SD & \\
\hline WMS-IV: LM I standard score $(n=47)$ & $6.20 \pm 1.81$ & $7.43 \pm 11.10$ & 0.730 \\
\hline WMS-IV: LM II standard score $(n=47)$ & $3.50 \pm 1.72$ & $4.43 \pm 3.48$ & 0.419 \\
\hline CVLT-II Short: total trials $1-4$ standard score $(n=46)$ & $33.70 \pm 8.60$ & $37.64 \pm 12.02$ & 0.339 \\
\hline CVLT-II Short: short-delay free recall standard score $(n=46)$ & $-1.75 \pm 0.89$ & $-1.19 \pm 1.26$ & 0.198 \\
\hline CVLT-II Short: long-delay free recall standard score $(n=46)$ & $-1.60 \pm 0.88$ & $-1.31 \pm 1.09$ & 0.437 \\
\hline CVLT-II Short: long-delay cued recall standard score $(n=46)$ & $-1.50 \pm 1.37$ & $-1.31 \pm 1.28$ & 0.677 \\
\hline CVLT-II Short: total intrusions standard score $(n=46)$ & $2.40 \pm 2.00$ & $1.88 \pm 1.78$ & 0.426 \\
\hline CVLT-II Short: recognition hits standard score $(n=45)$ & $-0.89 \pm 1.50$ & $-1.07 \pm 1.20$ & 0.703 \\
\hline CVLT-II Short: recognition FP standard score $(n=46)$ & $1.65 \pm 0.85$ & $2.01 \pm 1.78$ & 0.370 \\
\hline CDR-SOB (severity) $(n=84)$ & $5.25 \pm 2.62$ & $3.54 \pm 2.67$ & 0.023 \\
\hline
\end{tabular}

Social and cultural factors may play a role in how likely a patient is to create legal documents. All of the non-European patients included in our study were Indigenous. We found that patients of European ethnicity were more likely to have a Will, whereas patients of Indigenous background were less likely to have a Will. Indigenous people make up about $16 \%$ of the population of Saskatchewan, with about $65 \%$ identifying as First Nations, $33 \%$ as Metis, $0.2 \%$ as Inuit, and $1 \%$ as other or more than one Indigenous identity. ${ }^{48}$ Over half $(53 \%)$ of all First Nations people in Saskatchewan live on a reserve. ${ }^{48}$ Indigenous patients face special barriers to accessing the legal system. Common barriers include a lack of legal services within Indigenous communities, systemic racism, a distrust for the fairness of the legal system, and a lack of transportation to reach existing legal services. ${ }^{49}$ Furthermore, Indigenous populations live as the lowest economic group in Canada, adding an additional impediment to legal planning, given the high cost of most legal services. ${ }^{50}$ It should be noted that legal planning for Indigenous populations is a distinct topic and is beyond the scope of this discussion.
Having an Advanced Healthcare Directive was associated with factors that were not related to having a Will or Power of Attorney. Older patients were more likely to have an Advanced Healthcare Directive than younger patients. Impaired function and a high level of dependency as determined by the FAQ and BADLS were also significant. Similarly, patients with greater severity of cognitive and functional impairment were more likely to have an Advanced Healthcare Directive. Current housing type classified as "other," which included senior's centers, group homes, and special care homes, was also positively associated. These results are consistent with the well-known finding that many patients over age 65 experience increased medical morbidity and are less likely to live independently in the community. ${ }^{51}$ Living with multiple chronic conditions is associated with elevated risk of death, disability, and poor functional status. ${ }^{51}$ Consequently, these patients are more likely to express their medical wishes. There was likely a bias toward having an Advanced Healthcare Directive in patients living in an "other" housing category. Advanced Healthcare Directives are often 
Table 9: Neurological diagnosis of patients with and without an Advanced Healthcare Directive

\begin{tabular}{|c|c|c|c|c|}
\hline \multirow[b]{3}{*}{ Diagnosis } & \multicolumn{2}{|c|}{ Advanced Healthcare Directive } & \multirow[b]{2}{*}{ Total $(n=90)$} & \multirow[b]{3}{*}{$p$ value by category } \\
\hline & Yes $(n=20)$ & No $(n=70)$ & & \\
\hline & $n(\%)$ & $n(\%)$ & $n(\%)$ & \\
\hline Normal & $1(5.0)$ & $8(11.4)$ & $9(10.0)$ & 0.395 \\
\hline Mild cognitive impairment & $4(20.0)$ & $24(34.3)$ & $28(31.1)$ & 0.222 \\
\hline Frontotemporal dementia & $4(20.0)$ & $8(11.4)$ & $12(13.3)$ & 0.322 \\
\hline Alzheimer's disease & $9(45.0)$ & $25(35.7)$ & $34(37.8)$ & 0.447 \\
\hline Vascular dementia & $0(0.0)$ & $2(2.9)$ & $2(2.2)$ & 0.447 \\
\hline Dementia due to other etiologies & $2(10.0)$ & $3(4.3)$ & $5(5.6)$ & 0.327 \\
\hline
\end{tabular}

discussed with patients and families at the time of admission to long-term care and may even be required by some residences.

We anticipated that patients with worse scores on the MMSE and more advanced dementia would be more likely to have legal documents. Our findings, however, indicate no significant association between MMSE score or diagnosis and having any of the three legal documents. This raises concern because patients with poor cognition will have greater difficulty creating legal documents due to a potential lack of testamentary capacity. ${ }^{5}$ As there are no definitive predictors of who will develop dementia and who will not, it is important to create a Will, Power of Attorney, and Advanced Healthcare Directive when one is still in good health. ${ }^{21}$

Limitations to this study include a relatively small sample size and a cross-sectional study design where patient follow-up is not reported. Longitudinal follow-up would benefit future research in this area. Our study only included patients from rural areas. Access to lawyers is more difficult in rural centers compared to urban centers. For instance, there is only one private lawyer who serves the entire northern half of Saskatchewan. It would be interesting to compare data from our study with that of patients living in urban centers.

\section{Conclusion}

In this study, we observed a number of differences between patients who had a Will, Power of Attorney, and/or Advanced Healthcare Directive and those who did not. While there is no single indicator of having these documents, taking age, living situation, ethnicity, quality of life, functional status, and language difficulties into consideration may help to recognize patients who are at increased risk of leaving their legal affairs unattended to. Physicians who work with elderly populations, especially primary care physicians, can play an important role in enhancing patient access to the legal system. In particular, physicians can counsel patients who demonstrate risk factors for requiring further attention to legal planning. These risk factors may include being of older age, living at home with others, being of Indigenous background, having poor quality of life, having good functional status, and having good language fluency. By discussing legal documents early on with patients who might be at risk, we can better address challenges in legal and medical decision-making that arise when patients develop cognitive impairment.

\section{ACKNOWLedGements}

Funding from the Saskatchewan Ministry of Health supports clinical staff in the Rural and Remote Memory Clinic where the data were collected.

\section{Disclosures}

DM receives grants from the Canadian Institutes of Health Research (CIHR). SL, AK, EAK, CK, and MOC have nothing to disclose.

\section{Statement of Authorship}

SL wrote the manuscript. AK developed the research question, oversaw data collection, and assisted with manuscript preparation. EAK wrote sections of the manuscript pertaining to legal subject matter and assisted with manuscript preparation. CK performed the statistical analysis and assisted with manuscript preparation. MOC oversaw neuropsychological testing and assisted with manuscript preparation. DM oversaw data collection and assisted with manuscript preparation.

\section{REFERENCES}

1. Fiest KM, Jetté N, Roberts JI, et al. The prevalence and incidence of dementia: a systematic review and meta-analysis. Can J Neurol Sci. 2016;43(S1):S3-S50.

2. Charles L, Parmar J, Bremault-Phillips S, Dobbs B, Sacrey L, Sluggett B. Physician education on decision-making capacity assessment: current state and future directions. Can Fam Physician. 2017;63(1):e21-e30.

3. Alzheimer Society of Canada. Prevalence and monetary costs of dementia in Canada. Health Promot Chronic Dis Prev Can. 2016;36(10):231-2.

4. American Psychiatric Association. Diagnostic and statistical manual of mental disorders. 5th ed. Arlington, VA: American Psychiatric Publishing; 2013.

5. Voskou P, Douzenis A, Exonomou A, Papageorgiou SG. Testamentary capacity assessment: legal, medical, and neuropsychological issues. J Geriatr Psychiatry Neurol. 2018;31(1): 3-12.

6. Burla C, Rego G, Nunes R. Alzheimer, dementia and the living Will: a proposal. Med Health Care Philos. 2014;17(3):389-95.

7. The Wills Act of 1996, S. 21, Ch. W-14.1, Statutes of Saskatchewan (August 1, 1997).

8. Banks v. Goodfellow, L.R. 5, Q.B. 549 (1870).

9. Parker v. Felgate, L.R. 8, P.D. 171 (Eng P.D.A.) (1883). 
10. Intestate Succession Act of 1996, S. 6, Ch. I-13.1, Statutes of Saskatchewan (November 1, 1996).

11. Substitute Decisions Act of 1992, S. 7, S.46, Ch. 30, Statutes of Ontario (May 7, 2018).

12. Health Care Directives and Substitute Decision Health Care Decision Makers Act of 2015, S. 2(1)(b)-4, 16(1), 16(4), 17, Ch. H-0.002, Statutes of Saskatchewan (February 15, 2017).

13. Hewson T. Number of Canadians without Wills significantly under-reported. Canadian Legal Wills; 2016. Available at: https://www.legalWills.ca/blog/canadians-without-Wills/. Accessed March 21, 2018.

14. Lawyers Professional Indemnity Company (LAWPRO). Survey: more than half of Canadians do not have a signed Will. 2012. Available at: http://www.lawpro.ca/news/pdf/Wills-POAsurvey.pdf. Accessed March 21, 2018.

15. Canadian Hospice Palliative Care Association. Fact sheet: hospice palliative care in Canada. 2014. Available at: http://www.chpca.net/ media/330558/Fact_Sheet_HPC_in_CanadaSpring202014Final.pdf. Accessed March 21, 2018.

16. Elliot D. The age distribution of the Saskatchewan population. Sask Trends Monitor. 2012;24:2-10.

17. Laurent S. Rural Canada: access to health care. Ottawa: Parliamentary Research Branch; 2002.

18. Crossley M, Morgan D, Lanting S, Dal Bello-Haas V, Kirk A. Interdisciplinary research and interprofessional collaborative care in a memory clinic for rural and northern residents of western Canada: unique training ground for clinical psychology graduate students. Aust Psychol. 2008;43:231-8.

19. McEachern W, Kirk A, Morgan DG, Crossley M, Henry C. Reliability of the MMSE administered in-person and by telehealth. Can J Neurol Sci. 2008;35:643-6.

20. Morgan DG, Crossley M, Kirk A, et al. Improving access to dementia care: development and evaluation of a rural and remote memory clinic. Aging Mental Health. 2009;13:17-30.

21. Verity R, Kirk A, O'Connell ME, Karunanayake C, Morgan DG. The worried well? Characteristics of cognitively normal patients presenting to a rural and remote memory clinic. Can J Neurol Sci. 2018;45(2):158-67.

22. Verity R, Kirk A, Morgan D, Karunanayake C. Trends in medication use over 11 years in patients presenting to a rural and remote memory clinic. Can J Neurol Sci. 2016;43(6): 815-8.

23. Hager D, Kirk A, Morgan DG, Karunanayake C, O'Connell ME. Predictors of cognitive decline in a rural and remote Saskatchewan population with Alzheimer's disease. Am J Alzheimers Dis Other Demen. 2016;31(8):643-9.

24. Saleh S, Kirk A, Morgan DG, Karunanayake C. Less education predicts anticholinesterase discontinuation in dementia patients. Can J Neurol Sci. 2013;40(5):684-90.

25. Lacny C, Kirk A, Morgan DG, Karunanayake C. Predictors of cognitive impairment severity in rural patients at a memory clinic. Can J Neurol Sci. 2012;39(6):774-81.

26. Heggie M, Morgan D, Crossley M, et al. Quality of life in early dementia: comparison of rural patient and caregiver ratings at baseline and one year. Dementia. 2012;11(4):521-41.

27. Lacny C, Kirk A, Morgan DG, Karunanayake C. Does day length affect cognitive performance in memory clinic patients? Can J Neurol Sci. 2011;38(3):461-4.

28. Steve TA, Kirk A, Crossley M, et al. Medication use in patients presenting to a rural and remote memory clinic. Can J Neurol Sci. 2008;35(5):669-71.

29. Folstein MF, Folstein SE, McHugh PR. Mini-mental state: a practical method for grading the cognitive state of patients for the clinician. J Psychiatr Res. 1975;12(3):189-98.
30. Radloff LS. The CES-D scale: a self-report depression scale for research in the general population. Appl Psychol Meas. 1977; $1: 385-401$

31. Flanagan JC. A research approach to improving our quality of life. Am Psychol. 1978;33:138-47.

32. Hughes CP, Berg L, Danziger WL,Coben LA, Martin RL. A new clinical scale for the staging of dementia. Brit J Psychiatry. 1982;140:566-72.

33. Bucks RS, Ashworth DL, Wilcock GK, Siegfried K. Assessment of activities of daily living in dementia: development of the Bristol activities of daily living scale. Age Ageing. 1996;25:113-20.

34. Lawton MP, Brody EM. Assessment of older people: selfmaintaining and instrumental activities of daily living. Gerontologist. 1969;9(3):179-86.

35. Pfeffer RI, Kurosaki TT, Harrah CH Jr., Chance JM, Filos S. Measurement of functional activities in older adults in the community. J Gerontol. 1982;37(3):323-9.

36. Cummings JL, Mega M, Gray K, Rosenberg-Thompson S, Carusi DA, Gornbein J. The neuropsychiatric inventory: comprehensive assessment of psychopathology in dementia. Neurology. 1994;44(12):2308-14.

37. Delis DC, Kaplan E, Kramer JH. Delis-Kaplan executive function system: technical manual. San Antonio, TX: Harcourt Assessment Company; 2001.

38. Wechsler D. Administration and scoring manual for the Wechsler Memory Scale. 4th ed. San Antonio, TX: Pearson; 2009.

39. Delis DC, Kramer JH, Kaplan E, Ober BA. California verbal learning test: adult version manual. 2nd ed. San Antonio, TX: Psychological Corporation; 2000.

40. IBM Corporation. IBM SPSS statistics for Windows, version 24.0. Armonk, NY: IBM Corporation; 2016.

41. Fisk JE, Sharp CA. Age-related impairment in executive functioning: updating, inhibition, shifting, and access. J Clin Exp Neuropsychol. 2004;26(7):874-90.

42. Shao Z, Janse E, Visser K, Meyer AS. What do verbal fluency tasks measure? Predictors of verbal fluency performance in older adults. Front Psychol. 2014;5:772.

43. Burke DM, Shafto MA. Aging and language production. Curr Dir Psychol Sci. 2004;13(1):21-4.

44. Dixon RA, Hopp GA, Cohen AL, de Frias CM, Backman L. Selfreported memory compensation: similar patterns in Alzheimer's disease and very old adult samples. J Clin Exp Neuropsychol. 2003;25(3):382-90.

45. Perry-Young L, Owen G, Kelly S, Owens C. How people come to recognise a problem and seek medical help for a person showing early signs of dementia: a systematic review and meta-ethnography. Dementia. 2018;17(1):34-60.

46. Ahmed S, Mitchell J, Arnold R, Dawson K, Nestor PJ, Hodges JR. Memory complaints in mild cognitive impairment, worried well, and semantic dementia patients. Alzheimer Dis Assoc Disord. 2008;22(3):227-35.

47. Salthouse TA. What cognitive abilities are involved in trail-making performance? Intelligence. 2011;39(4):222-32.

48. Aboriginal peoples: a fact sheet for Saskatchewan. Statistics Canada; 2016. Available at: http://www.statcan.gc.ca/pub/89656-x/89-656-x2016009-eng.htm. Accessed May 12, 2018.

49. Wright AC. Access to justice in indigenous communities: an intercultural strategy to improve access to justice. Canadian Research Institute for Law and the Family; 2017. Available at: http://www.crilf.ca/Documents/AccesstoJusticeinIndigenous Communities-May2017.pdf. Accessed May 12, 2018.

50. Morse BW. Native people and legal services in Canada. McGill Law J. 1976;22:517.

51. Salive ME. Multimorbidity in older adults. Epidemiol Rev. 2013;35:75-83. 